\title{
Lamellar Assembly of Conical Molecules Possessing a Fullerene Apex in Crystals and Liquid Crystals
}

\author{
Yu-Wu Zhong, Yutaka Matsuo,* and Eiichi Nakamura*
}

Nakamura Functional Carbon Cluster Project, ERATO, Japan Science and Technology

Agency (JST), The University of Tokyo, Hongo, Bunkyo-ku, Tokyo 113-0033, Japan

E-mail: matsuo@chem.s.u-tokyo.ac.jp; nakamura@chem.s.u-tokyo.ac.jp

Table of Contents

1. Synthetic Procedures

2. Single Crystal X-ray Crystallographic Analysis of $\mathbf{1}$ and $\mathbf{2}$

3. Optical Textures of Liquid Crystals

4. DSC Measurement of Liquid Crystals

5. X-ray Diffraction Data for Liquid Crystals 


\section{Synthetic Procedures}

General. All experiments were carried out under argon using standard Schlenk techniques. THF was distilled from $\mathrm{Na} / \mathrm{K}$ alloy, and dichloromethane was distilled from $\mathrm{CaH}_{2}$ before use. 4- $\mathrm{BrC}_{6} \mathrm{H}_{4} \mathrm{C} \equiv \mathrm{CSiMe}_{3},{ }^{n} \mathrm{C}_{4} \mathrm{H}_{9} \mathrm{SiMe}_{2} \mathrm{Cl},{ }^{n} \mathrm{C}_{8} \mathrm{H}_{17} \mathrm{SiMe}_{2} \mathrm{Cl},{ }^{n} \mathrm{C}_{10} \mathrm{H}_{21} \mathrm{SiMe}_{2} \mathrm{Cl}$, and ${ }^{n} \mathrm{C}_{12} \mathrm{H}_{25} \mathrm{SiMe}_{2} \mathrm{Cl}$ were commercially available and used as received. ${ }^{n} \mathrm{C}_{14} \mathrm{H}_{29} \mathrm{SiMe}_{2} \mathrm{Cl}$ was prepared according to literature. ${ }^{1}$ All ${ }^{1} \mathrm{H}(500 \mathrm{MHz})$ and ${ }^{13} \mathrm{C}\left\{{ }^{1} \mathrm{H}\right\}(125 \mathrm{MHz})$ spectra were recorded on JEOL ECA500 spectrometers. Spectra were reported in parts per million from internal tetramethylsilane $(\delta 0.00 \mathrm{ppm})$ or residual protons of the deuterated solvent for ${ }^{1} \mathrm{H}$ NMR, from solvent carbon (e.g. $\delta 77.00 \mathrm{ppm}$ for chloroform) for ${ }^{13} \mathrm{C}\left\{{ }^{1} \mathrm{H}\right\}$ NMR. HPLC analyses were performed on Shimadzu LC-10A system equipped with SPD-M10A diode array detector and Cosmosil-Buckyprep column (4.6 x $250 \mathrm{~mm}$, Nacalai Tesque Co.). Preparative HPLC separations were performed by the use of a Buckyprep column (20 mm x $250 \mathrm{~mm}$ ) using toluene/2-propanol (6/4) as eluent. High-resolution mass spectra were measured on a JEOL JMS-T100LC ESI-TOF mass spectrometer. IR spectra were recorded on Applied Systems Inc. React-IR 1000.

\section{A General Procedure for the Preparation of $4-\mathrm{BrC}_{6} \mathrm{H}_{4} \mathrm{C} \equiv \mathrm{CSiMe}_{2}{ }^{n} \mathrm{C}_{n} \mathrm{H}_{2 n+1}$. To a} solution of 1-bromo-4-ethylnylbenzene $(362 \mathrm{mg}, 2.0 \mathrm{mmol})$ in $10 \mathrm{~mL}$ of THF was added dropwise a solution of $i-\operatorname{PrMgBr}(2.1 \mathrm{mmol})$ in THF $(3.1 \mathrm{~mL}, 0.67 \mathrm{M})$ at $0{ }^{\circ} \mathrm{C}$. The mixture was stirred for $20 \mathrm{~min}$ before the ice bath was removed. Stirring was continued for another $10 \mathrm{~min}$ at room temperature, followed by the addition of 2.5 mmol of corresponding $n-\mathrm{C}_{\mathrm{n}} \mathrm{H}_{2 \mathrm{n}+1} \mathrm{SiMe}_{2} \mathrm{Cl}$. Two hours later, the reaction was quenched by $5.0 \mathrm{~mL}$ of saturated aqueous ammonium chloride solution. After extraction with ethyl acetate, the combined organic phase was concentrated and subjected to flash column chromatography to afford the desired product.

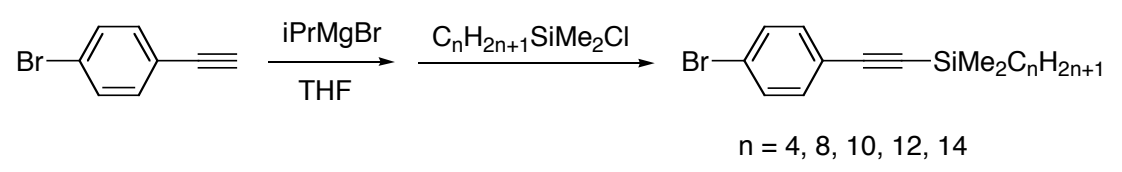

1-(4-Bromophenylethynyl)- ${ }^{n}$ butyl-dimethylsilane $\quad\left[4-\mathrm{Br}_{-} \mathrm{C}_{6} \mathrm{H}_{4} \mathrm{C} \equiv \mathrm{CSiMe}_{2}\left(n-\mathrm{C}_{4} \mathrm{H}_{9}\right)\right]$. ${ }^{1} \mathrm{H} \mathrm{NMR}\left(\mathrm{CDCl}_{3}\right): \delta 0.21\left(\mathrm{~s}, 6 \mathrm{H}, \mathrm{SiMe}_{2}\right), 0.68\left(\mathrm{t}, J=8.00 \mathrm{~Hz}, 2 \mathrm{H}, \mathrm{SiCH}_{2}\right), 0.91(\mathrm{t}, J=$

1 Itami, K.; Terakawa, K.; Yoshida, J.-I.; Kajimoto, O. J. Am. Chem. Soc. 2003, 125, 6058. 
$\left.6.90 \mathrm{~Hz}, 3 \mathrm{H}, \mathrm{CH}_{2} \mathrm{CH}_{3}\right), 1.37-1.41\left(\mathrm{~m}, 4 \mathrm{H}, 2 \mathrm{CH}_{2}\right), 7.32(\mathrm{~d}, J=8.60 \mathrm{~Hz}, 2 \mathrm{H}, \mathrm{ArH}), 7.42$ $(\mathrm{d}, J=8.60 \mathrm{~Hz}, 2 \mathrm{H}, \mathrm{ArH}) .{ }^{13} \mathrm{C} \mathrm{NMR}\left(\mathrm{CDCl}_{3}\right): \delta-1.79\left(2 \mathrm{C}, \mathrm{SiMe}_{2}\right), 13.81\left(\mathrm{SiCH}_{2}\right)$,

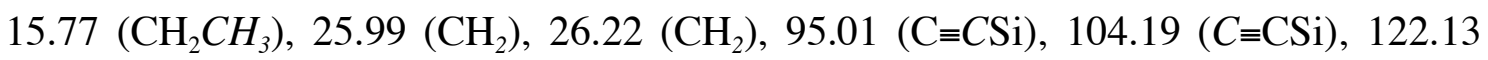
(1C, Ar), 122.67 (1C, Ar), 131.42 (2C, Ar), 133.35 (2C, Ar).

1-(4-Bromophenylethynyl)-dimethyl- ${ }^{n}$ octylsilane $\quad\left[4-\mathrm{Br}_{-} \mathrm{C}_{6} \mathrm{H}_{4} \mathrm{C} \equiv \mathrm{CSiMe}_{2}\left(n-\mathrm{C}_{8} \mathrm{H}_{17}\right)\right]$. ${ }^{1} \mathrm{H} \mathrm{NMR}\left(\mathrm{CDCl}_{3}\right): \delta 0.22\left(\mathrm{~s}, 6 \mathrm{H}, \mathrm{SiMe}_{2}\right), 0.69$ (t, $\left.J=8.10 \mathrm{~Hz}, 2 \mathrm{H}, \mathrm{SiCH}_{2}\right), 0.89$ (t, $J=$ $\left.6.90 \mathrm{~Hz}, 3 \mathrm{H}, \mathrm{CH}_{2} \mathrm{CH}_{3}\right), 1.37-1.41\left(\mathrm{~m}, 12 \mathrm{H}, 6 \mathrm{CH}_{2}\right), 7.32$ (d, $\left.J=8.60 \mathrm{~Hz}, 2 \mathrm{H}, \mathrm{ArH}\right), 7.42$ $(\mathrm{d}, J=8.00 \mathrm{~Hz}, 2 \mathrm{H}, \mathrm{ArH}) .{ }^{13} \mathrm{C} \mathrm{NMR}\left(\mathrm{CDCl}_{3}\right): \delta-1.78\left(2 \mathrm{C}, \mathrm{SiMe}_{2}\right), 14.11\left(\mathrm{SiCH}_{2}\right)$, $16.05\left(\mathrm{CH}_{2} \mathrm{CH}_{3}\right), 22.69\left(\mathrm{CH}_{2}\right), 23.79\left(\mathrm{CH}_{2}\right), 29.26\left(\mathrm{CH}_{2}\right), 29.30\left(\mathrm{CH}_{2}\right), 31.93\left(\mathrm{CH}_{2}\right)$,

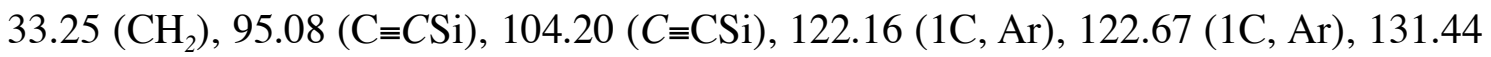
(2C, Ar), 133.37 (2C, Ar).

1-(4-Bromophenylethynyl)-dimethyl- ${ }^{n}$ decylsilane $\left[4-\mathrm{Br}^{-\mathrm{C}_{6}} \mathrm{H}_{4} \mathrm{C} \equiv \mathrm{CSiMe}_{2}\left(n-\mathrm{C}_{10} \mathrm{H}_{21}\right)\right]$. ${ }^{1} \mathrm{H} \mathrm{NMR}\left(\mathrm{CDCl}_{3}\right): \delta 0.21\left(\mathrm{~s}, 6 \mathrm{H}, \mathrm{SiMe}_{2}\right), 0.68\left(\mathrm{t}, J=8.00 \mathrm{~Hz}, 2 \mathrm{H}, \mathrm{SiCH}_{2}\right), 0.88(\mathrm{t}, J=$ $\left.6.90 \mathrm{~Hz}, 3 \mathrm{H}, \mathrm{CH}_{2} \mathrm{CH}_{3}\right), 1.25-1.43\left(\mathrm{~m}, 16 \mathrm{H}, 8 \mathrm{CH}_{2}\right), 7.31$ (d, J=8.55 Hz, 2H, ArH), 7.42 $(\mathrm{d}, J=8.60 \mathrm{~Hz}, 2 \mathrm{H}, \mathrm{ArH}) .{ }^{13} \mathrm{C} \mathrm{NMR}\left(\mathrm{CDCl}_{3}\right): \delta-1.67\left(2 \mathrm{C}, \mathrm{SiMe}_{2}\right), 14.23\left(\mathrm{SiCH}_{2}\right)$, $16.14\left(\mathrm{CH}_{2} \mathrm{CH}_{3}\right), 22.80\left(\mathrm{CH}_{2}\right), 23.88\left(\mathrm{CH}_{2}\right), 29.44\left(\mathrm{CH}_{2}\right), 29.46\left(\mathrm{CH}_{2}\right), 29.71\left(\mathrm{CH}_{2}\right)$, $29.78\left(\mathrm{CH}_{2}\right), 32.02\left(\mathrm{CH}_{2}\right), 33.35\left(\mathrm{CH}_{2}\right), 95.19(\mathrm{C} \equiv C \mathrm{CSi}), 104.28(C \equiv \mathrm{CSi}), 122.25(1 \mathrm{C}$, Ar), 122.77 (1C, Ar), 131.53 (2C, Ar), 133.48 (2C, Ar).

\section{1-(4-Bromophenylethynyl)-dimethyl- ${ }^{n}$ dodecylsilane}

[4-Br- $\mathbf{C}_{\mathbf{6}} \mathbf{H}_{\mathbf{4}} \mathbf{C} \equiv \mathrm{CSiMe}_{\mathbf{2}}\left(\boldsymbol{n}-\mathbf{C}_{\mathbf{1 2}} \mathbf{H}_{\mathbf{2 5}}\right)$ ]. ${ }^{1} \mathrm{H} \mathrm{NMR}\left(\mathrm{CDCl}_{3}\right): \delta 0.21\left(\mathrm{~s}, 6 \mathrm{H}, \mathrm{SiMe}_{2}\right), 0.68(\mathrm{t}, J=$ $\left.8.00 \mathrm{~Hz}, 2 \mathrm{H}, \mathrm{SiCH}_{2}\right), 0.88\left(\mathrm{t}, J=6.90 \mathrm{~Hz}, 3 \mathrm{H}, \mathrm{CH}_{2} \mathrm{CH}_{3}\right), 1.25-1.42\left(\mathrm{~m}, 20 \mathrm{H}, 10 \mathrm{CH}_{2}\right)$, $7.32(\mathrm{~d}, J=8.60 \mathrm{~Hz}, 2 \mathrm{H}, \mathrm{ArH}), 7.42(\mathrm{~d}, J=8.60 \mathrm{~Hz}, 2 \mathrm{H}, \mathrm{ArH}) \cdot{ }^{13} \mathrm{C} \mathrm{NMR}\left(\mathrm{CDCl}_{3}\right): \delta$ -1.77 (2C, $\left.\mathrm{SiMe}_{2}\right), 14.13\left(1 \mathrm{C}, \mathrm{SiCH}_{2}\right), 16.04\left(1 \mathrm{C}, \mathrm{CH}_{2} \mathrm{CH}_{3}\right), 22.70\left(1 \mathrm{C}, \mathrm{CH}_{2}\right), 23.78(1 \mathrm{C}$, $\left.\mathrm{CH}_{2}\right), 29.35\left(1 \mathrm{C}, \mathrm{CH}_{2}\right), 29.38\left(1 \mathrm{C}, \mathrm{CH}_{2}\right), 29.61\left(1 \mathrm{C}, \mathrm{CH}_{2}\right), 29.67\left(1 \mathrm{C}, \mathrm{CH}_{2}\right), 29.73(2 \mathrm{C}$, $\left.2 \mathrm{CH}_{2}\right), 31.93\left(1 \mathrm{C}, \mathrm{CH}_{2}\right), 33.25\left(1 \mathrm{C}, \mathrm{CH}_{2}\right), 95.05(\mathrm{C} \equiv C \mathrm{Si}), 104.20(C \equiv \mathrm{CSi}), 122.16(1 \mathrm{C}$, Ar), 122.66 (1C, Ar), 131.42 (2C, Ar), 133.36 (2C, Ar).

\section{1-(4-Bromophenylethynyl)-dimethyl- ${ }^{n}$ tetradecylsilane}

[4-Br- $\mathbf{C}_{\mathbf{6}} \mathbf{H}_{\mathbf{4}} \mathbf{C} \equiv \mathrm{CSiMe}_{\mathbf{2}}\left(\boldsymbol{n}-\mathbf{C}_{\mathbf{1 4}} \mathbf{H}_{\mathbf{2} 9}\right)$ ]. ${ }^{1} \mathrm{H} \mathrm{NMR}\left(\mathrm{CDCl}_{3}\right): \delta 0.22\left(\mathrm{~s}, 6 \mathrm{H}, \mathrm{SiMe}_{2}\right), 0.68(\mathrm{t}, J=$ $\left.8.00 \mathrm{~Hz}, 2 \mathrm{H}, \mathrm{SiCH}_{2}\right), 0.89\left(\mathrm{t}, J=6.90 \mathrm{~Hz}, 3 \mathrm{H}, \mathrm{CH}_{2} \mathrm{CH}_{3}\right), 1.25-1.42\left(\mathrm{~m}, 24 \mathrm{H}, 12 \mathrm{CH}_{2}\right)$, $7.32(\mathrm{~d}, J=8.60 \mathrm{~Hz}, 2 \mathrm{H}, \mathrm{ArH}), 7.43(\mathrm{~d}, J=8.05 \mathrm{~Hz}, 2 \mathrm{H}, \mathrm{ArH}) \cdot{ }^{13} \mathrm{C} \mathrm{NMR}\left(\mathrm{CDCl}_{3}\right): \delta$ 
-1.78 (2C, $\left.\mathrm{SiMe}_{2}\right), 14.12\left(1 \mathrm{C}, \mathrm{SiCH}_{2}\right), 16.05\left(1 \mathrm{C}, \mathrm{CH}_{2} \mathrm{CH}_{3}\right), 22.70\left(1 \mathrm{C}, \mathrm{CH}_{2}\right), 23.79(1 \mathrm{C}$, $\left.\mathrm{CH}_{2}\right), 29.35\left(1 \mathrm{C}, \mathrm{CH}_{2}\right), 29.38\left(1 \mathrm{C}, \mathrm{CH}_{2}\right), 29.60\left(2 \mathrm{C}, 2 \mathrm{CH}_{2}\right), 29.67\left(2 \mathrm{C}, 2 \mathrm{CH}_{2}\right), 29.71$

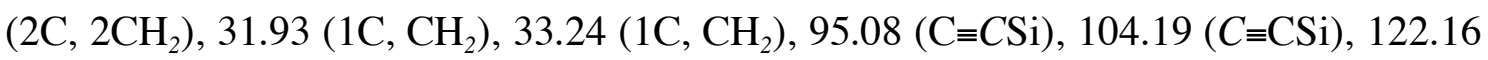
(1C, Ar), 122.66 (1C, Ar), 131.43 (2C, Ar), 133.36 (2C, Ar).

\section{(4-Bromophenylethynyl)-dimethyl-(7-tridecyl)silane}

\{4-Br- $\left.\mathrm{C}_{6} \mathrm{H}_{4} \mathrm{C} \equiv \mathrm{CSSiMe}_{2}\left[\mathrm{CH}\left(\boldsymbol{n}-\mathrm{C}_{6} \mathbf{H}_{13}\right)_{2}\right]\right\}$. To a solution of dichlorodimethylsilane (0.65 $\mathrm{mL}, 5.0 \mathrm{mmol}$ ) in $10 \mathrm{~mL}$ of THF at $0{ }^{\circ} \mathrm{C}$ was added dropwise $2.0 \mathrm{mmol}$ of a Grignard reagent prepared from 7-bromotridecane $(660 \mathrm{mg}, 2.5 \mathrm{mmol})$ and magnesium turnings (60 mg, $2.5 \mathrm{mmol}$ ) in $7 \mathrm{~mL}$ of $\mathrm{THF}$ at $70{ }^{\circ} \mathrm{C}$. After stirring overnight at room temperature, the mixture was concentrated under vacuum and dissolved in $5 \mathrm{~mL}$ of THF again. This solution was then added dropwise to another solution of 4-bromophenyl alkylnyl magnesium bromide in $5 \mathrm{~mL}$ of THF, which was prepared in situ from 1-bromo-4-ethylnylbenzene $(360 \mathrm{mg}, 2.0 \mathrm{mmol})$ and $i$-PrMgBr $(2.9 \mathrm{~mL}$ of 0.70 M THF solution, $2.0 \mathrm{mmol}$ ). The mixture was stirred at room temperature for 4 days. The reaction was then quenched by $5.0 \mathrm{~mL}$ of saturated aqueous ammonium chloride solution. After extraction with ethyl acetate, the combined organic phase was concentrated and subjected to flash column chromatography to afford the desired product (180 $\mathrm{mg}, 21 \%)$ and the unreacted starting material 1-bromo-4-ethylnylbenzene (140 mg, 40\%). ${ }^{1} \mathrm{H}$ NMR $\left(\mathrm{CDCl}_{3}\right): \delta 0.21$ (s, 6H, $\left.\mathrm{SiMe}_{2}\right), 0.75$ (m, 1H, SiCH), 0.88 (t, $J$ $\left.=6.90 \mathrm{~Hz}, 6 \mathrm{H}, 2 \mathrm{CH}_{2} \mathrm{CH}_{3}\right), 1.29-1.55\left(\mathrm{~m}, 20 \mathrm{H}, 10 \mathrm{CH}_{2}\right), 7.30(\mathrm{~d}, J=8.00 \mathrm{~Hz}, 2 \mathrm{H}, \mathrm{ArH})$, $7.43(\mathrm{~d}, J=8.05 \mathrm{~Hz}, 2 \mathrm{H}, \mathrm{ArH}) .{ }^{13} \mathrm{C} \mathrm{NMR}\left(\mathrm{CDCl}_{3}\right): \delta-2.33\left(2 \mathrm{C}, \mathrm{SiMe}_{2}\right), 14.11(2 \mathrm{C})$, $22.72\left(2 \mathrm{C}, 2 \mathrm{CH}_{2} \mathrm{CH}_{3}\right), 25.17(1 \mathrm{C}), 28.94\left(2 \mathrm{C}, 2 \mathrm{CH}_{2}\right), 29.73\left(4 \mathrm{C}, 4 \mathrm{CH}_{2}\right), 31.85(2 \mathrm{C}$, $\left.2 \mathrm{CH}_{2}\right), 95.34(\mathrm{C} \equiv C \mathrm{CSi}), 104.37$ ( $\left.\mathrm{C} \equiv \mathrm{CSi}\right), 122.33,122.58,131.42$ (2C), 133.30 (2C).

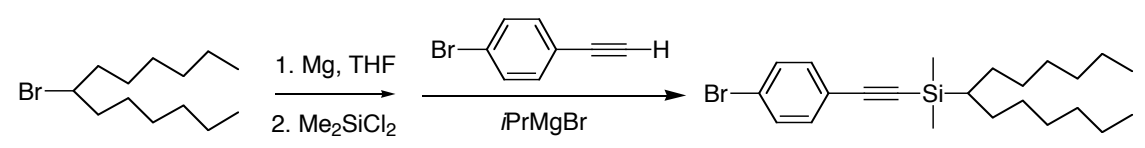

\section{A General Procedure for the Penta-addition to $\mathbf{C}_{60}$. A solution of} 4-bromophenylethynyl trimethylsilane ( $\mathrm{R}=\mathrm{Me}, 607 \mathrm{mg}, 2.4 \mathrm{mmol})$ in $8 \mathrm{~mL}$ of $\mathrm{THF}$ was added to magnesium turnings $(60 \mathrm{mg}, 2.5 \mathrm{mmol})$ in $2 \mathrm{~mL}$ of THF. The mixture was stirred at room temperature for 3 hours to give the corresponding Grignard reagent, to which $512 \mathrm{mg}$ ( $2.5 \mathrm{mmol}$ ) of copper bromide dimethyl sulfide complex was added. 10 min later, a solution of $\mathrm{C}_{60}(144 \mathrm{mg}, 0.2 \mathrm{mmol})$ in $10 \mathrm{~mL}$ of $o$-dichlorobenzene was 
added in one portion. After reaction for 2 hours at room temperature, $1.24 \mathrm{~mL}$ (20 mmol) of iodomethane was then added. The reaction was quenched by $0.1 \mathrm{~mL}$ of a saturated aqueous ammonium chloride solution after stirring for another 3 hours. Toluene $(20 \mathrm{~mL})$ was added, and the mixture was filtered through a pad of silica gel. The concentrated residue was purified by flash column chromatography to give $290 \mathrm{mg}$ of $\mathbf{1}$ in a yield of $90 \%$. The molecules 2-7 were synthesized in the same way from the corresponding phenyl bromide prepared above.

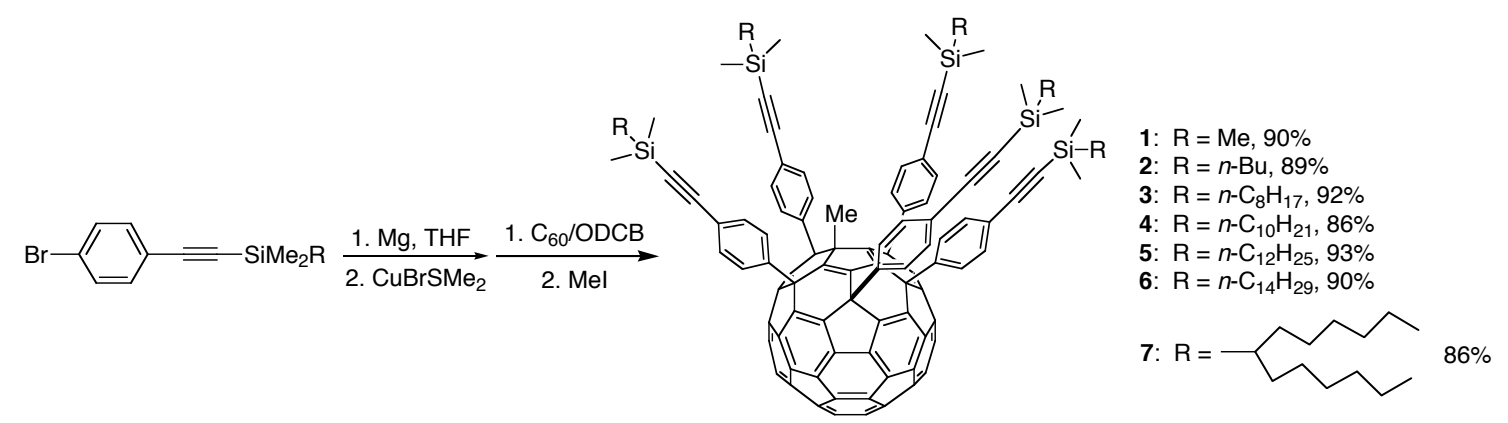

6,9,12,15,18-Pentakis(4-trimethylsilylethynylphenyl)-1-methyl-1,6,9,12,15,18-hexah $\operatorname{ydro}\left(\mathbf{C}_{\mathbf{6 0}}-\mathbf{I}_{\mathrm{h}}\right)[\mathbf{5 , 6}]$ fullerene (1). ${ }^{1} \mathrm{H} \mathrm{NMR}\left(\mathrm{CDCl}_{3}\right): \delta 0.21\left(\mathrm{~s}, 9 \mathrm{H}, \mathrm{SiMe}_{3}\right), 0.28(\mathrm{~s}, 18 \mathrm{H}$, $\mathrm{SiMe}_{3}$ ), 0.30 (s, 18H, $\mathrm{SiMe}_{3}$ ), 1.36 (s, 3H, C $\left.60 \mathrm{Me}\right), 7.07$ (d, J = 8.00 Hz, 2H, ArH), 7.20 $(\mathrm{d}, J=8.00 \mathrm{~Hz}, 2 \mathrm{H}, \mathrm{ArH}), 7.44$ (d, $J=8.00 \mathrm{~Hz}, 4 \mathrm{H}, \operatorname{ArH}), 7.46(\mathrm{~d}, J=8.00 \mathrm{~Hz}, 4 \mathrm{H}$, $\operatorname{ArH}), 7.60(\mathrm{~d}, J=8.00 \mathrm{~Hz}, 4 \mathrm{H}, \operatorname{ArH}), 7.71(\mathrm{~d}, J=8.00 \mathrm{~Hz}, 4 \mathrm{H}, \operatorname{ArH}) .{ }^{13} \mathrm{C} \mathrm{NMR}$ $\left(\mathrm{CDCl}_{3}\right): \delta-0.17\left(3 \mathrm{C}, \mathrm{SiMe}_{3}\right),-0.06\left(6 \mathrm{C}, 2 \mathrm{SiMe}_{3}\right),-0.02\left(6 \mathrm{C}, 2 \mathrm{SiMe}_{3}\right), 34.35\left(\mathrm{C}_{60} \mathrm{Me}\right)$, $57.95\left(2 \mathrm{C}, 2 \mathrm{C}_{60}\left(\mathrm{C}_{\alpha}\right)\right), 60.81\left(2 \mathrm{C}, 2 \mathrm{C}_{60}\left(\mathrm{C}_{\alpha}\right)\right), 62.22\left(1 \mathrm{C}, \mathrm{C}_{60}\left(\mathrm{C}_{\alpha}\right)\right), 62.33\left(1 \mathrm{C}, \mathrm{C}_{60}(\mathrm{CMe})\right)$, $95.35(1 \mathrm{C}, \mathrm{C} \equiv C \mathrm{CSi}), 95.45$ (2C, $2 \mathrm{C} \equiv C \mathrm{CSi}), 95.47$ (2C, $2 \mathrm{C} \equiv C \mathrm{Si}), 104.07$ (1C, $C \equiv \mathrm{CSi})$, 104.36 (2C, $2 C \equiv \mathrm{CSi}$ ), 104.51 (2C, 2C $\equiv \mathrm{CSi}$ ), 122.04 (1C, Ar), 122.79 (2C, Ar), 122.95 (2C, Ar), 127.92 (4C, Ar), 128.46 (4C, Ar), 129.54 (2C, Ar), 131.78 (2C, Ar), 132.34 (4C, Ar), 132.62 (4C, Ar), 137.93, 139.58, 142.44, 142.48, 143.50, 143.66, 143.92, 144.12 , 144.30, 144.35, 144.45, 144.61, 145.25, 145.47, 147.10, 147.28, 147.30, 147.82, 148.19, 148.26, 148.37, 148.47, 148.71, 148.75, 148.82, 151.31, 152.37, 156.61, 160.64. APCI-MS (-): m/z 1600 [M- . APCI-HRMS (-): calcd for $\mathrm{C}_{116} \mathrm{H}_{68} \mathrm{Si}_{5}\left[\mathrm{M}^{-}\right]$1600.4167, found 1600.4129 .

6,9,12,15,18-Pentakis(4-" butyldimethylsilylethynylphenyl)-1-methyl-1,6,9,12,15,18hexahydro $\left(\mathbf{C}_{60}-I_{\mathrm{h}}\right)[5,6]$ fullerene (2). ${ }^{1} \mathrm{H} \mathrm{NMR}\left(\mathrm{CDCl}_{3}\right): \delta 0.16\left(\mathrm{~s}, 6 \mathrm{H}, \mathrm{SiMe}_{2}\right), 0.25(\mathrm{~s}$, $\left.12 \mathrm{H}, 2 \mathrm{SiMe}_{2}\right), 0.28\left(\mathrm{~s}, 12 \mathrm{H}, 2 \mathrm{SiMe}_{2}\right), 0.63\left(\mathrm{t}, J=8.00 \mathrm{~Hz}, 2 \mathrm{H}, \mathrm{SiCH}_{2}\right), 0.73(\mathrm{~m}, 8 \mathrm{H}$, 
$\left.4 \mathrm{SiCH}_{2}\right), 0.93\left(\mathrm{~m}, 15 \mathrm{H}, 5 \mathrm{CH}_{2} \mathrm{CH}_{3}\right), 1.34-1.47\left(\mathrm{~m}, 20 \mathrm{H}, 10 \mathrm{CH}_{2}\right), 1.45\left(\mathrm{~s}, 3 \mathrm{H}, \mathrm{C}_{60} \mathrm{Me}\right.$, overlapped), $7.08(\mathrm{~d}, J=8.60 \mathrm{~Hz}, 2 \mathrm{H}), 7.20(\mathrm{~d}, J=8.00 \mathrm{~Hz}, 2 \mathrm{H}), 7.46(\mathrm{~m}, 8 \mathrm{H}), 7.60(\mathrm{~d}$, $J=8.00 \mathrm{~Hz}, 4 \mathrm{H}), 7.72(\mathrm{~d}, J=8.60 \mathrm{~Hz}, 4 \mathrm{H}) .{ }^{13} \mathrm{C} \mathrm{NMR}\left(\mathrm{CDCl}_{3}\right): \delta-1.79\left(2 \mathrm{C}, \mathrm{SiMe}_{2}\right)$, $-1.73\left(4 \mathrm{C}, 2 \mathrm{SiMe}_{2}\right),-1.70\left(4 \mathrm{C}, 2 \mathrm{SiMe}_{2}\right), 13.84\left(5 \mathrm{C}, 5 \mathrm{SiCH}_{2}\right), 15.78\left(5 \mathrm{C}, 5 \mathrm{CH}_{2} \mathrm{CH}_{3}\right)$, $26.03\left(5 \mathrm{C}, 5 \mathrm{CH}_{2}\right), 26.26\left(5 \mathrm{C}, 5 \mathrm{CH}_{2}\right), 34.36\left(\mathrm{C}_{60} M e\right), 57.96\left(2 \mathrm{C}, 2 \mathrm{C}_{60}\left(\mathrm{sp}^{3}\right)\right), 60.82(2 \mathrm{C}$, $\left.2 \mathrm{C}_{60}\left(\mathrm{sp}^{3}\right)\right), 62.25\left(1 \mathrm{C}, \mathrm{C}_{60}\left(\mathrm{sp}^{3}\right)\right), 62.32\left(1 \mathrm{C}, \mathrm{C}_{60}(C) \mathrm{Me}\right), 94.84(1 \mathrm{C}, \mathrm{C} \equiv C \mathrm{Si}), 94.96(2 \mathrm{C}$,

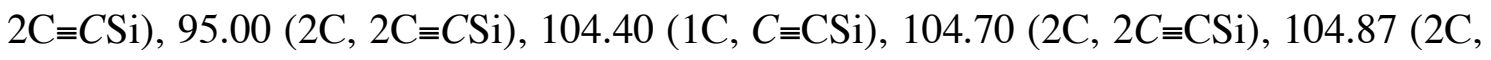
$2 C \equiv \mathrm{CSi}), 122.13,122.88,123.04,127.93,128.47,129.56,131.82,132.37,132.66$, $137.90,139.55,142.46,142.85,143.50,143.66,143.91$, 144.12, 144.30, 144.36, 144.45, 144.60, 145.25, 145.48, 147.11, 147.29, 147.31, 147.83, 148.20, 148.26, 148.38, 148.48, $148.71,148.76,148.83,151.30,152.41,156.65,160.06$. APCI-HRMS (-): calcd for $\mathrm{C}_{131} \mathrm{H}_{98} \mathrm{Si}_{5}\left[\mathrm{M}^{-}\right]$1810.6515, found 1810.6476 .

\section{6,9,12,15,18-Pentakis(4-" octyldimethylsilylethynylphenyl)-1-methyl-1,6,9,12,15,18-} hexahydro $\left(\mathbf{C}_{60}-I_{\mathrm{h}}\right)\left[\mathbf{5 , 6 ] f u l l e r e n e ~ ( 3 ) . ~}{ }^{1} \mathrm{H} \mathrm{NMR}\left(\mathrm{CDCl}_{3}\right): \delta 0.17\left(\mathrm{~s}, 6 \mathrm{H}, \mathrm{SiMe}_{2}\right), 0.25(\mathrm{~s}\right.$, $\left.12 \mathrm{H}, 2 \mathrm{SiMe}_{2}\right), 0.28\left(\mathrm{~s}, 12 \mathrm{H}, 2 \mathrm{SiMe}_{2}\right), 0.63\left(\mathrm{t}, J=8.60 \mathrm{~Hz}, 2 \mathrm{H}, \mathrm{SiCH}_{2}\right), 0.74(\mathrm{~m}, 8 \mathrm{H}$, $\left.4 \mathrm{SiCH}_{2}\right), 0.87$ (m, $\left.15 \mathrm{H}, 5 \mathrm{CH}_{2} \mathrm{CH}_{3}\right), 1.20-1.50$ (m, 63H, $\mathrm{C}_{60} \mathrm{Me}+30 \mathrm{CH}_{2}$, overlapped), $7.09(\mathrm{~d}, J=8.60 \mathrm{~Hz}, 2 \mathrm{H}), 7.21(\mathrm{~d}, J=8.05 \mathrm{~Hz}, 2 \mathrm{H}), 7.44(\mathrm{~d}, J=8.00 \mathrm{~Hz}, 4 \mathrm{H}), 7.46$ (d, $J=8.05 \mathrm{~Hz}, 4 \mathrm{H}), 7.60(\mathrm{~d}, J=8.05 \mathrm{~Hz}, 4 \mathrm{H}), 7.72(\mathrm{~d}, J=8.60 \mathrm{~Hz}, 4 \mathrm{H}) .{ }^{13} \mathrm{C} \mathrm{NMR}$ $\left(\mathrm{CDCl}_{3}\right): \delta-1.77\left(2 \mathrm{C}, \mathrm{SiMe}_{2}\right),-1.72\left(4 \mathrm{C}, 2 \mathrm{SiMe}_{2}\right),-1.69\left(4 \mathrm{C}, 2 \mathrm{SiMe}_{2}\right), 14.12(5 \mathrm{C}$, $\left.5 \mathrm{SiCH}_{2}\right), 16.03\left(1 \mathrm{C}, \mathrm{CH}_{2} \mathrm{CH}_{3}\right), 16.11\left(2 \mathrm{C}, 2 \mathrm{CH}_{2} \mathrm{CH}_{3}\right), 16.13\left(2 \mathrm{C}, 2 \mathrm{CH}_{2} \mathrm{CH}_{3}\right), 22.66(1 \mathrm{C}$, $\left.\mathrm{CH}_{2}\right), 22.69\left(4 \mathrm{C}, 4 \mathrm{CH}_{2}\right), 23.72\left(1 \mathrm{C}, \mathrm{CH}_{2}\right), 23.79\left(4 \mathrm{C}, 4 \mathrm{CH}_{2}\right), 29.27\left(10 \mathrm{C}, 10 \mathrm{CH}_{2}\right), 31.89$ $\left(1 \mathrm{C}, \mathrm{CH}_{2}\right), 31.93\left(4 \mathrm{C}, 4 \mathrm{CH}_{2}\right), 33.23\left(1 \mathrm{C}, \mathrm{CH}_{2}\right), 33.29\left(4 \mathrm{C}, 4 \mathrm{CH}_{2}\right), 34.30\left(\mathrm{C}_{60} \mathrm{Me}\right), 57.96$ $\left(2 \mathrm{C}, 2 \mathrm{C}_{60}\left(\mathrm{sp}^{3}\right)\right), 60.83\left(2 \mathrm{C}, 2 \mathrm{C}_{60}\left(\mathrm{sp}^{3}\right)\right), 62.25\left(1 \mathrm{C}, \mathrm{C}_{60}\left(\mathrm{sp}^{3}\right)\right), 62.32\left(1 \mathrm{C}, \mathrm{C}_{60}(C) \mathrm{Me}\right), 94.86$ $(1 \mathrm{C}, \mathrm{C} \equiv C \mathrm{CSi}), 95.00(4 \mathrm{C}, 4 \mathrm{C} \equiv C \mathrm{CSi}), 104.40$ (1C,C $\equiv \mathrm{CSi}), 104.70(2 \mathrm{C}, 2 C \equiv \mathrm{CSi}), 104.86$ (2C, $2 C \equiv \mathrm{CSi}), 123.14,122.90,123.06,127.90,128.43$, 129.55, 131.81, 132.37, 132.65, $137.91,139.56,142.46,142.48,142.85,143.50,143.65,143.91,144.12,144.30,144.36$, 144.46, 144.60, 145.25, 145.48, 147.10, 147.29, 247.31, 147.83, 148.19, 148.27, 148.37, $148.48,148.72,148.77,148.83,151.32,152.41,156.63,160.10$. APCI-HRMS (-): calcd for $\mathrm{C}_{151} \mathrm{H}_{138} \mathrm{Si}_{5}\left[\mathrm{M}^{-}\right]$2090.9645, found 2090.9682 .

6,9,12,15,18-Pentakis(4- ${ }^{n}$ decyldimethylsilylethynylphenyl)-1-methyl-1,6,9,12,15,18hexahydro $\left(\mathbf{C}_{60}-I_{\mathrm{h}}\right)[5,6]$ fullerene (4). ${ }^{1} \mathrm{H} \mathrm{NMR}\left(\mathrm{CDCl}_{3}\right): \delta 0.16\left(\mathrm{~s}, 6 \mathrm{H}, \mathrm{SiMe}_{2}\right), 0.25(\mathrm{~s}$, $\left.12 \mathrm{H}, 2 \mathrm{SiMe}_{2}\right), 0.27\left(\mathrm{~s}, 12 \mathrm{H}, 2 \mathrm{SiMe}_{2}\right), 0.64\left(\mathrm{t}, J=8.00 \mathrm{~Hz}, 2 \mathrm{H}, \mathrm{SiCH}_{2}\right), 0.72(\mathrm{~m}, 8 \mathrm{H}$, 
$\left.4 \mathrm{SiCH}_{2}\right), 0.87\left(\mathrm{~m}, 15 \mathrm{H}, 5 \mathrm{CH}_{2} \mathrm{CH}_{3}\right), 1.24-1.48\left(\mathrm{~m}, 83 \mathrm{H}, \mathrm{C}_{60} \mathrm{Me}+40 \mathrm{CH}_{2}\right.$, overlapped), $7.08(\mathrm{~d}, J=8.00 \mathrm{~Hz}, 2 \mathrm{H}), 7.20(\mathrm{~d}, J=8.00 \mathrm{~Hz}, 2 \mathrm{H}), 7.44$ (d, $J=8.05 \mathrm{~Hz}, 4 \mathrm{H}), 7.46$ (d, $J=8.00 \mathrm{~Hz}, 4 \mathrm{H}), 7.60(\mathrm{~d}, J=8.00 \mathrm{~Hz}, 4 \mathrm{H}), 7.71(\mathrm{~d}, J=8.00 \mathrm{~Hz}, 4 \mathrm{H}) .{ }^{13} \mathrm{C} \mathrm{NMR}$ $\left(\mathrm{CDCl}_{3}\right): \delta-1.80\left(2 \mathrm{C}, \mathrm{SiMe}_{2}\right),-1.73\left(4 \mathrm{C}, 2 \mathrm{SiMe}_{2}\right),-1.70\left(4 \mathrm{C}, 2 \mathrm{SiMe}_{2}\right), 14.12(5 \mathrm{C}$, $\left.5 \mathrm{SiCH}_{2}\right), 16.04\left(1 \mathrm{C}, \mathrm{CH}_{2} \mathrm{CH}_{3}\right), 16.10\left(2 \mathrm{C}, 2 \mathrm{CH}_{2} \mathrm{CH}_{3}\right), 16.12\left(2 \mathrm{C}, 2 \mathrm{CH}_{2} \mathrm{CH}_{3}\right), 22.69$ (5C, $\left.5 \mathrm{CH}_{2}\right), 23.72\left(1 \mathrm{C}, \mathrm{CH}_{2}\right), 23.79\left(4 \mathrm{C}, 4 \mathrm{CH}_{2}\right), 29.33\left(\mathrm{~m}, 10 \mathrm{C}, 10 \mathrm{CH}_{2}\right), 29.63(\mathrm{~m}, 10 \mathrm{C}$, $\left.10 \mathrm{CH}_{2}\right), 31.91\left(5 \mathrm{C}, 5 \mathrm{CH}_{2}\right), 33.26\left(1 \mathrm{C}, \mathrm{CH}_{2}\right), 33.29\left(4 \mathrm{C}, 4 \mathrm{CH}_{2}\right), 34.30\left(\mathrm{C}_{60} \mathrm{Me}\right), 57.95$ $\left(2 \mathrm{C}, 2 \mathrm{C}_{60}\left(\mathrm{sp}^{3}\right)\right), 60.81\left(2 \mathrm{C}, 2 \mathrm{C}_{60}\left(\mathrm{sp}^{3}\right)\right), 62.23\left(1 \mathrm{C}, \mathrm{C}_{60}\left(\mathrm{sp}^{3}\right)\right), 62.31\left(1 \mathrm{C}, \mathrm{C}_{60}(C) \mathrm{Me}\right), 94.86$

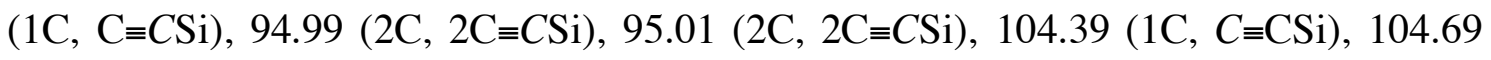
(2C, $2 C \equiv \mathrm{CSi}), 104.85$ (2C, $2 \mathrm{C} \equiv \mathrm{CSi}), 122.13,122.89,123.05,127.89,128.42,129.54$, 131.81, 132.36, 132.64, 137.89, 139.55, 142.45, 142.84, 143.48, 143.64, 143.90, 144.11, 144.29, 144.34, 144.43, 144.59, 145.24, 145.47, 147.10, 147.28, 147.30, 147.82, 148.18, $148.25,148.36,148.47,148.70,148.76,148.81,151.30,152.40,156.62,160.08$. APCI-HRMS (-): calcd for $\mathrm{C}_{161} \mathrm{H}_{159} \mathrm{Si}_{5}\left[\mathrm{M}^{-}+\mathrm{H}\right] 2232.1288$, found 2232.1246.

\section{6,9,12,15,18-Pentakis(4- ${ }^{n}$ dodecyldimethylsilylethynylphenyl)-1-methyl-1,6,9,12,15,1}

8-hexahydro $\left(\mathbf{C}_{60}-I_{\mathrm{h}}\right)\left[\mathbf{5 , 6 ] f u l l e r e n e ~ ( 5 ) . ~}{ }^{1} \mathrm{H} \mathrm{NMR}\left(\mathrm{CDCl}_{3}\right): \delta 0.17\left(\mathrm{~s}, 6 \mathrm{H}, \mathrm{SiMe}_{2}\right), 0.25(\mathrm{~s}\right.$, $\left.12 \mathrm{H}, 2 \mathrm{SiMe}_{2}\right), 0.28\left(\mathrm{~s}, 12 \mathrm{H}, 2 \mathrm{SiMe}_{2}\right), 0.64\left(\mathrm{t}, J=8.00 \mathrm{~Hz}, 2 \mathrm{H}, \mathrm{SiCH}_{2}\right), 0.74(\mathrm{~m}, 8 \mathrm{H}$, $4 \mathrm{SiCH}_{2}$ ), 0.87 (m, 15H, 5 $\left.\mathrm{CH}_{2} \mathrm{CH}_{3}\right), 1.24-1.48$ (m, 103H, $\mathrm{C}_{60} \mathrm{Me}+50 \mathrm{CH}_{2}$, overlapped), $7.08(\mathrm{~d}, J=8.55 \mathrm{~Hz}, 2 \mathrm{H}), 7.20(\mathrm{~d}, J=8.55 \mathrm{~Hz}, 2 \mathrm{H}), 7.44$ (d, $J=8.00 \mathrm{~Hz}, 4 \mathrm{H}), 7.46$ (d, $J=8.00 \mathrm{~Hz}, 4 \mathrm{H}), 7.60(\mathrm{~d}, J=8.60 \mathrm{~Hz}, 4 \mathrm{H}), 7.71(\mathrm{~d}, J=8.00 \mathrm{~Hz}, 4 \mathrm{H}) .{ }^{13} \mathrm{C} \mathrm{NMR}$ $\left(\mathrm{CDCl}_{3}\right): \delta-1.79\left(2 \mathrm{C}, \mathrm{SiMe}_{2}\right),-1.71\left(4 \mathrm{C}, 2 \mathrm{SiMe}_{2}\right),-1.68\left(4 \mathrm{C}, 2 \mathrm{SiMe}_{2}\right), 14.13(5 \mathrm{C}$, $\left.5 \mathrm{SiCH}_{2}\right), 16.06\left(1 \mathrm{C}, \mathrm{CH}_{2} \mathrm{CH}_{3}\right), 16.13\left(2 \mathrm{C}, 2 \mathrm{CH}_{2} \mathrm{CH}_{3}\right), 16.15\left(2 \mathrm{C}, 2 \mathrm{CH}_{2} \mathrm{CH}_{3}\right), 22.69$ (5C, $\left.5 \mathrm{CH}_{2}\right), 23.74\left(1 \mathrm{C}, \mathrm{CH}_{2}\right), 23.80\left(4 \mathrm{C}, 4 \mathrm{CH}_{2}\right), 29.36\left(10 \mathrm{C}, 10 \mathrm{CH}_{2}\right), 29.70\left(\mathrm{~m}, 20 \mathrm{C}, 20 \mathrm{CH}_{2}\right)$, $31.92\left(5 \mathrm{C}, 5 \mathrm{CH}_{2}\right), 33.28\left(1 \mathrm{C}, \mathrm{CH}_{2}\right), 33.31\left(4 \mathrm{C}, 4 \mathrm{CH}_{2}\right), 34.30\left(\mathrm{C}_{60} \mathrm{Me}\right), 57.96(2 \mathrm{C}$, $\left.2 \mathrm{C}_{60}\left(\mathrm{sp}^{3}\right)\right), 60.83\left(2 \mathrm{C}, 2 \mathrm{C}_{60}\left(\mathrm{sp}^{3}\right)\right), 62.25\left(1 \mathrm{C}, \mathrm{C}_{60}\left(\mathrm{sp}^{3}\right)\right), 62.33\left(1 \mathrm{C}, \mathrm{C}_{60}(C) \mathrm{Me}\right), 94.86(1 \mathrm{C}$, $\mathrm{C} \equiv C \mathrm{Si}), 95.00(2 \mathrm{C}, 2 \mathrm{C} \equiv C \mathrm{Si}), 95.02$ (2C, $2 \mathrm{C} \equiv C \mathrm{Si}), 104.42$ (1C, $C \equiv \mathrm{CSi}), 104.72$ (2C, $2 C \equiv \mathrm{CSi}), 104.88$ (2C, $2 C \equiv \mathrm{CSi}), 122.16,122.91,123.08,127.91,128.43,129.55,131.82$, 132.39, 132.66, 137.92, 139.57, 142.45, 142.48, 142.85, 143.48, 143.64, 143.90, 144.11, $144.29,144.35,144.44,144.59,145.23$, 145.46, 147.10, 147.28, 147.30, 147.82, 148.18 , 148.26, 148.36, 148.47, 148.70, 148.75, 148.81, 151.31, 152.40, 156.62, 160.10 . APCI-MS (-): $m / z 2371$ [M- $]$ APCI-HRMS (-): calcd for $\mathrm{C}_{171} \mathrm{H}_{177} \mathrm{Si}_{5}\left[\mathrm{M}^{-}-\mathrm{H}\right]$ 2370.2697 , found 2370.2653 . 


\section{6,9,12,15,18-Pentakis (4-" ${ }^{n}$ tetradecyldimethylsilylethynylphenyl)-1-methyl-1,6,9,12,1}

5,18-hexahydro $\left(\mathbf{C}_{60}-I_{\mathbf{h}}\right)\left[\mathbf{5 , 6 ] f u l l e r e n e ~ ( 6 ) . ~}{ }^{1} \mathrm{H} \mathrm{NMR}\left(\mathrm{CDCl}_{3}\right): \delta 0.19\left(\mathrm{~s}, 6 \mathrm{H}, \mathrm{SiMe}_{2}\right)\right.$, $0.27\left(\mathrm{~s}, 12 \mathrm{H}, 2 \mathrm{SiMe}_{2}\right), 0.30\left(\mathrm{~s}, 12 \mathrm{H}, 2 \mathrm{SiMe}_{2}\right), 0.66$ (t, $\left.J=8.00 \mathrm{~Hz}, 2 \mathrm{H}, \mathrm{SiCH}_{2}\right), 0.74$ (m, $\left.8 \mathrm{H}, 4 \mathrm{SiCH}_{2}\right), 0.89\left(\mathrm{~m}, 15 \mathrm{H}, 5 \mathrm{CH}_{2} \mathrm{CH}_{3}\right), 1.24-1.48\left(\mathrm{~m}, 123 \mathrm{H}, \mathrm{C}_{60} \mathrm{Me}+60 \mathrm{CH}_{2}\right.$, overlapped), 7.10 (d, $J=8.05 \mathrm{~Hz}, 2 \mathrm{H}), 7.22$ (d, $J=8.05 \mathrm{~Hz}, 2 \mathrm{H}), 7.45$ (d, $J=8.00 \mathrm{~Hz}$, 4H), $7.48(\mathrm{~d}, J=8.00 \mathrm{~Hz}, 4 \mathrm{H}), 7.62(\mathrm{~d}, J=8.00 \mathrm{~Hz}, 4 \mathrm{H}), 7.73(\mathrm{~d}, J=8.00 \mathrm{~Hz}, 4 \mathrm{H}) .{ }^{13} \mathrm{C}$ NMR $\left(\mathrm{CDCl}_{3}\right): \delta-1.77\left(2 \mathrm{C}, \mathrm{SiMe}_{2}\right),-1.70\left(4 \mathrm{C}, 2 \mathrm{SiMe}_{2}\right),-1.67$ (4C, $\left.2 \mathrm{SiMe}_{2}\right), 14.14(5 \mathrm{C}$, $\left.5 \mathrm{SiCH}_{2}\right), 16.08\left(1 \mathrm{C}, \mathrm{CH}_{2} \mathrm{CH}_{3}\right), 16.16\left(4 \mathrm{C}, 4 \mathrm{CH}_{2} \mathrm{CH}_{3}\right), 22.70\left(5 \mathrm{C}, 5 \mathrm{CH}_{2}\right), 23.76(1 \mathrm{C}$, $\left.\mathrm{CH}_{2}\right), 23.82\left(4 \mathrm{C}, 4 \mathrm{CH}_{2}\right), 29.37\left(10 \mathrm{C}, 10 \mathrm{CH}_{2}\right), 29.70\left(\mathrm{~m}, 30 \mathrm{C}, 30 \mathrm{CH}_{2}\right), 31.93\left(5 \mathrm{C}, 5 \mathrm{CH}_{2}\right)$, $33.30\left(1 \mathrm{C}, \mathrm{CH}_{2}\right), 33.33\left(4 \mathrm{C}, 4 \mathrm{CH}_{2}\right), 34.30\left(\mathrm{C}_{60} \mathrm{Me}\right), 57.95\left(2 \mathrm{C}, 2 \mathrm{C}_{60}\left(\mathrm{sp}^{3}\right)\right), 60.81(2 \mathrm{C}$, $\left.2 \mathrm{C}_{60}\left(\mathrm{sp}^{3}\right)\right), 62.23\left(1 \mathrm{C}, \mathrm{C}_{60}\left(\mathrm{sp}^{3}\right)\right), 62.31\left(1 \mathrm{C}, \mathrm{C}_{60}(C) \mathrm{Me}\right), 94.86(1 \mathrm{C}, \mathrm{C} \equiv C \mathrm{Si}), 94.99(2 \mathrm{C}$, $2 \mathrm{C} \equiv C \mathrm{Si}), 95.00(2 \mathrm{C}, 2 \mathrm{C} \equiv C \mathrm{Si}), 104.45$ (1C, $C \equiv \mathrm{CSi}), 104.74$ (2C, $2 \mathrm{C} \equiv \mathrm{CSi}), 104.90$ (2C, $2 C \equiv \mathrm{CSi}), 122.16,122.91,123.08,127.67,127.91,128.43,129.54,130.52,131.84$, 132.40, 132.67, 137.93, 139.57, 142.43, 142.47, 142.83, 143.45, 143.61, 143.87, 144.09, 144.27, 144.33, 144.43, 144.56, 145.20, 145.42, 147.06, 147.24, 147.27, 147.78, 148.15, $148.22,148.33,148.43,148.67,148.72,148.78,151.29,152.36,156.59,160.10$. APCI-MS (-): m/z 2511 [M']. APCI-HRMS (-): calcd for $\mathrm{C}_{161} \mathrm{H}_{159} \mathrm{Si}_{5}\left[\mathrm{M}^{-}\right] 2511.4340$, found 2511.4225 .

\section{6,9,12,15,18-Pentakis[4-(7-tridecyl)dimethylsilylethynylphenyl]-1-methyl-1,6,9,12,1} 5,18-hexahydro $\left(\mathbf{C}_{60}-I_{\mathbf{h}}\right)\left[\mathbf{5 , 6 ] f u l l e r e n e ~ ( 7 ) . ~}{ }^{1} \mathrm{H} \mathrm{NMR}\left(\mathrm{CDCl}_{3}\right): \delta 0.15\left(\mathrm{~s}, 6 \mathrm{H}, \mathrm{SiMe}_{2}\right)\right.$, $0.24\left(\mathrm{~s}, 12 \mathrm{H}, 2 \mathrm{SiMe}_{2}\right), 0.26$ (s, $\left.12 \mathrm{H}, 2 \mathrm{SiMe}_{2}\right), 0.77-0.87$ (m, 35H, $5 \mathrm{SiCH}, 10 \mathrm{CH}_{2} \mathrm{CH}_{3}$, overlapped), 1.24-1.56 (m, $103 \mathrm{H}, \mathrm{C}_{60} \mathrm{Me}+50 \mathrm{CH}_{2}$, overlapped), 7.09 (d, $J=8.00 \mathrm{~Hz}$, 2H), $7.19(\mathrm{~d}, J=8.60 \mathrm{~Hz}, 2 \mathrm{H}), 7.42(\mathrm{~d}, J=8.60 \mathrm{~Hz}, 4 \mathrm{H}), 7.45(\mathrm{~d}, J=8.60 \mathrm{~Hz}, 4 \mathrm{H})$, $7.60(\mathrm{~d}, J=8.60 \mathrm{~Hz}, 4 \mathrm{H}), 7.71(\mathrm{~d}, J=8.60 \mathrm{~Hz}, 4 \mathrm{H}) .{ }^{13} \mathrm{C} \mathrm{NMR}\left(\mathrm{CDCl}_{3}\right): \delta-2.30(2 \mathrm{C}$, $\mathrm{SiMe}_{2}$ ), -2.25 (8C, 4SiMe $), 14.14$ (10C), 22.69 (10C), 25.08 (1C), 25.14 (2C), 25.17 (2C), 28.95 (m, 10C), $26.96(\mathrm{~m}, 20 \mathrm{C}), 31.82$ (10C), $34.28\left(\mathrm{C}_{60} \mathrm{Me}\right), 57.96(2 \mathrm{C}$, $\left.2 \mathrm{C}_{60}\left(\mathrm{sp}^{3}\right)\right), 60.83\left(2 \mathrm{C}, 2 \mathrm{C}_{60}\left(\mathrm{sp}^{3}\right)\right), 62.26\left(1 \mathrm{C}, \mathrm{C}_{60}\left(\mathrm{sp}^{3}\right)\right), 62.32\left(1 \mathrm{C}, \mathrm{C}_{60}(C) \mathrm{Me}\right), 95.06(1 \mathrm{C}$, $\mathrm{C} \equiv C \mathrm{Si}), 95.22$ (4C, $4 \mathrm{C} \equiv C \mathrm{Si}), 104.57$ (1C, $C \equiv \mathrm{CSi}), 104.86$ (2C, $2 C \equiv \mathrm{CSi}), 105.01$ (2C, $2 C \equiv \mathrm{CSi}), 122,33,123.08,123.25,127.89,128.42,129.58,131.75,132.30,132.58$, $137.87,139.51,142.45,142.47,142.84,143.49,143.63$, 143.89, 144.10, 144.29, 144.35, $144.45,144.58,145.23,145.47,147.11,147.28,147.31$, 147.82, 148.18, 148.26, 148.37, $148.47,148.70,148.76,148.81,151.32,152.44,156.65,160.16$. APCI-MS (-): $m / z$ 2441 [M- $\mathrm{M}^{-}$. APCI-HRMS (-): calcd for $\mathrm{C}_{176} \mathrm{H}_{188} \mathrm{Si}_{5}\left[\mathrm{M}^{-}\right]$2441.3557, found 2441.3517. 


\section{Single Crystal X-ray Crystallographic Analysis of 1 and 2}

Single crystals of $\mathbf{1}$ and $\mathbf{2}$ suitable for X-ray diffraction studies were mounted on a MacScience DIP2030 Imaging Plate diffractometer using MoKo (graphite monochromated, $\lambda=0.71073 \AA$ ) radiation. Crystal data and data statistics are summarized in Table S1. The structures of $\mathbf{1} \cdot(\text { toluene) })_{2}$ and 2 were solved by the directed method (SHELXS-97). The positional and thermal parameters of non-hydrogen atoms were refined anisotropically on $F^{2}$ by the full-matrix least-squares method, using SHELXL-97. Parameters of butyl carbon atoms in $\mathbf{2}$ were refined isotropically with restraints on the bond lengths and angles, due to fluctuation of the butyl groups. Hydrogen atoms were placed at calculated positions and refined with riding mode on their corresponding carbon atoms. In the subsequent refinement, the function $\Sigma \omega\left(F_{\mathrm{o}}^{2}-F_{\mathrm{c}}{ }^{2}\right)^{2}$ was minimized, where $\left|F_{\mathrm{o}}\right|$ and $\left|F_{\mathrm{c}}\right|$ are the observed and calculated structure factor amplitudes, respectively. The agreement indices are defined as $R 1=\Sigma$ $\left(\left\|F_{\mathrm{o}}|-| F_{\mathrm{c}}\right\|\right) / \Sigma\left|F_{\mathrm{o}}\right|$ and $\mathrm{w} R 2=\left[\Sigma \omega\left(F_{\mathrm{o}}{ }^{2}-F_{\mathrm{c}}{ }^{2}\right)^{2} / \Sigma\left(\omega F_{\mathrm{o}}{ }^{4}\right)\right]^{1 / 2}$. Both crystals contain disorder problems in the five side chains and solvent molecules. This is the reason of high $\mathrm{R}$ values and low data completeness.

(a)

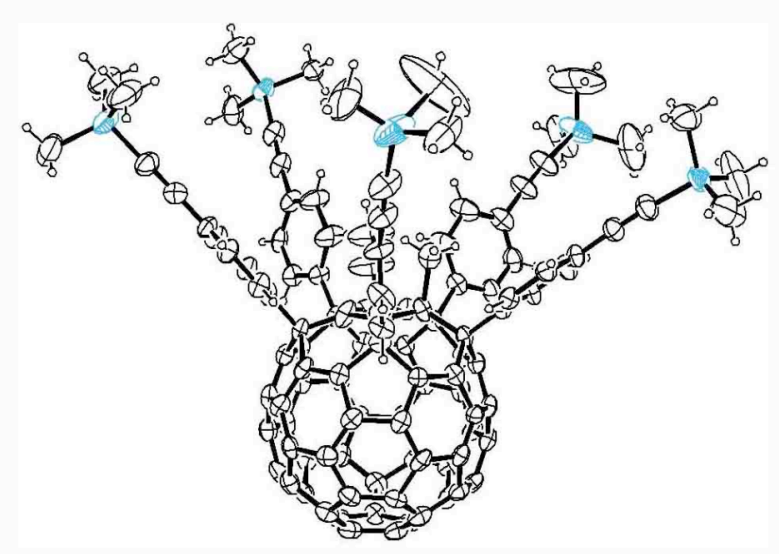

(b)

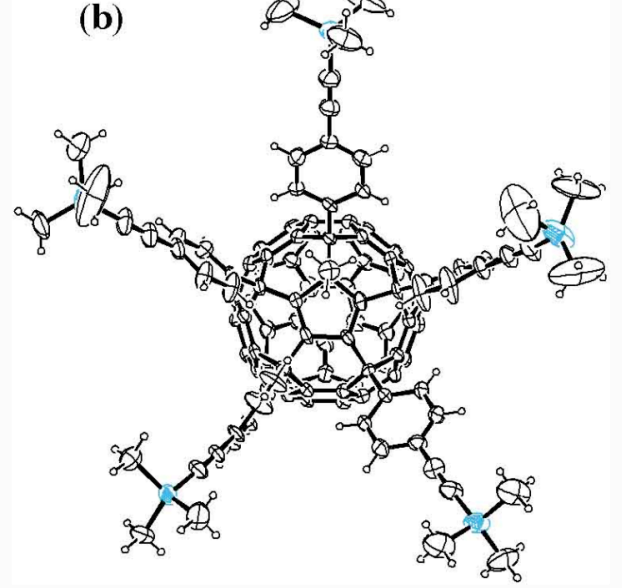

Figure S1. Molecular structure of 1. (a) Side view. (b) Top view. 


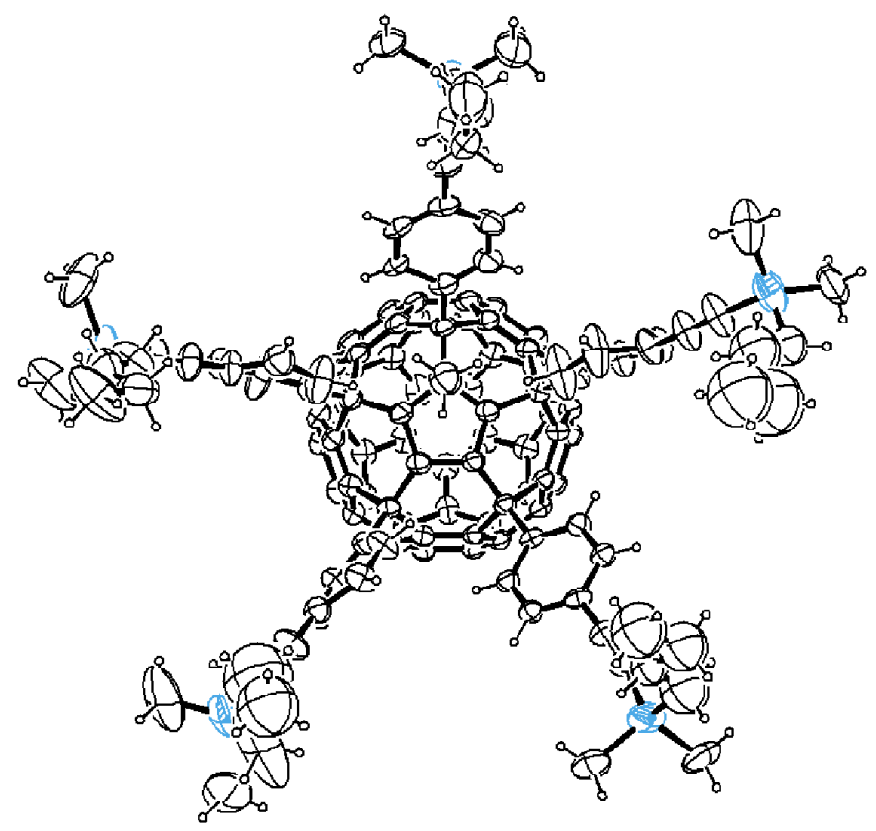

Figure S2. Molecular structure of 2.

Table S1. Crystal data and data collection parameters for $\mathbf{1}$ and 2.

\begin{tabular}{|c|c|c|}
\hline & $\mathbf{1} \cdot(\text { toluene })_{2}$ & 2 \\
\hline crystal system & triclinic & monoclinic \\
\hline space group & $P-1$ (No. 2) & $P 2_{1} / n$ (No. 14$)$ \\
\hline$a, \AA$ & $14.449(3)$ & $18.546(2)$ \\
\hline$b, \AA$ & $17.506(6)$ & $17.643(2)$ \\
\hline$c, \AA$ & $23.235(7)$ & $32.427(2)$ \\
\hline$\alpha$, degree & $103.57(1)$ & 90 \\
\hline$\beta$, degree & $100.73(2)$ & $100.441(6)$ \\
\hline$\gamma$, degree & $105.18(2)$ & 90 \\
\hline$V, \AA^{3}$ & 5318 & 10434.7 \\
\hline$Z$ & 2 & 4 \\
\hline$T, \mathrm{~K}$ & $153(2)$ & $153(2)$ \\
\hline crystal size, mm & $0.32 \times 0.22 \times 0.13$ & $0.75 \times 0.30 \times 0.12$ \\
\hline $2 \theta_{\min }, 2 \theta_{\max }$, degree & $4.52,51.54$ & $4.42,51.54$ \\
\hline no. of refl. (unique) & 16452 & 19418 \\
\hline no. of refl. $(I>2 \sigma(I))$ & 6676 & 8741 \\
\hline no. of parameters & 1135 & 1206 \\
\hline$R 1, w R 2$ (all data) & $0.296,0.484$ & $0.258,0.501$ \\
\hline$R, w R(I>2 \sigma(I))$ & $0.178,0.418$ & $0.182,0.443$ \\
\hline GOF on $F^{2}$ & 1.304 & 1.744 \\
\hline$\Delta, \mathrm{e} \AA^{-3}$ & $1.08,-0.49$ & $1.11,-0.66$ \\
\hline
\end{tabular}




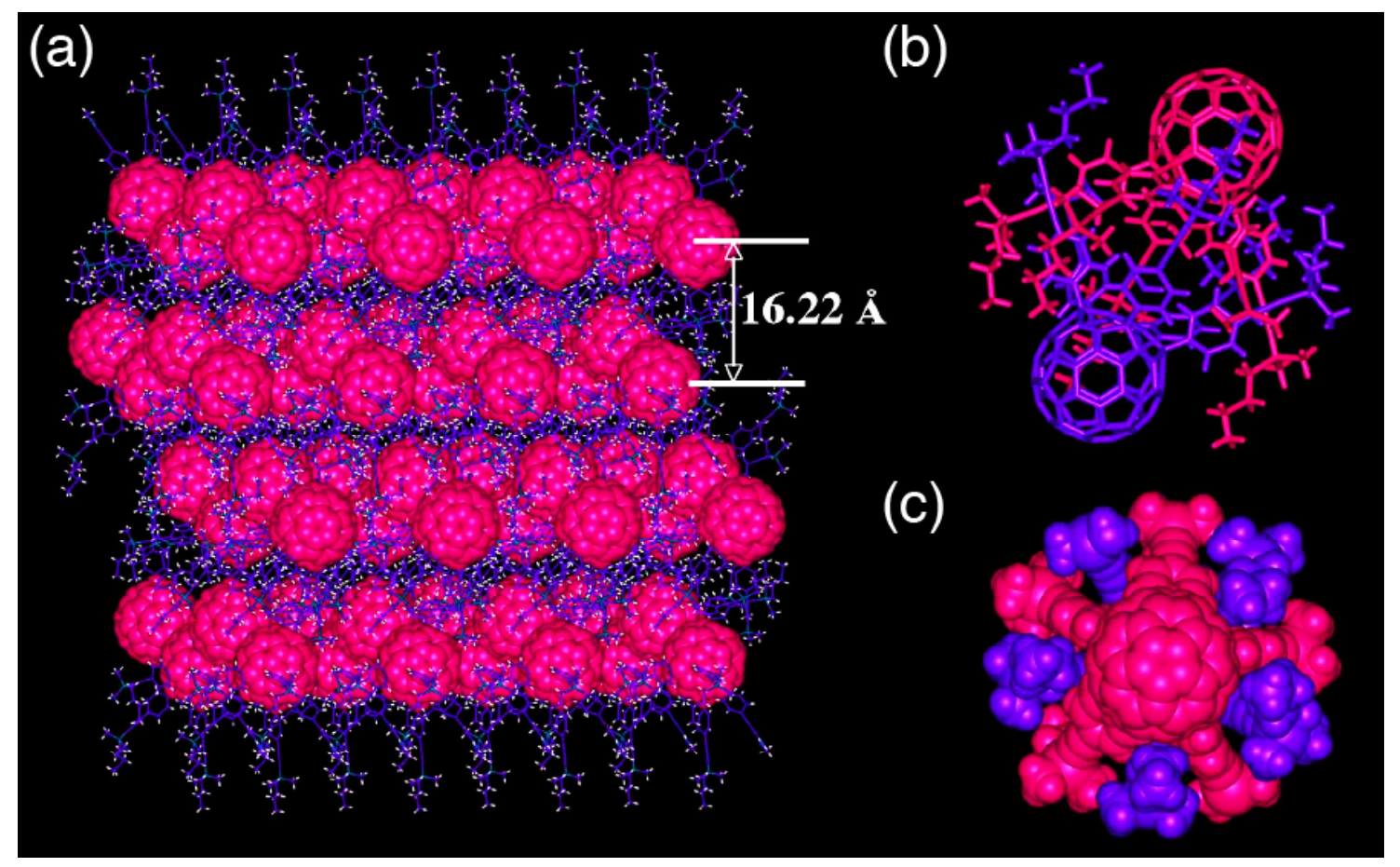

Figure S3. (a) Layered crystal packing of 2. View along the (120) axis. (b) Side view of an interdigitated dimer of $\mathbf{2}$. Each molecule serves as a part of the neighboring layer. (c) Top view of the interdigitated dimer.

Lamellar organization. The large cavity in crystal structure in $\mathbf{1}$ was filled with solvent. In the liquid crystalline compound $\mathbf{4}$, it was filled with alkyl groups and they have similar interlayer distance.

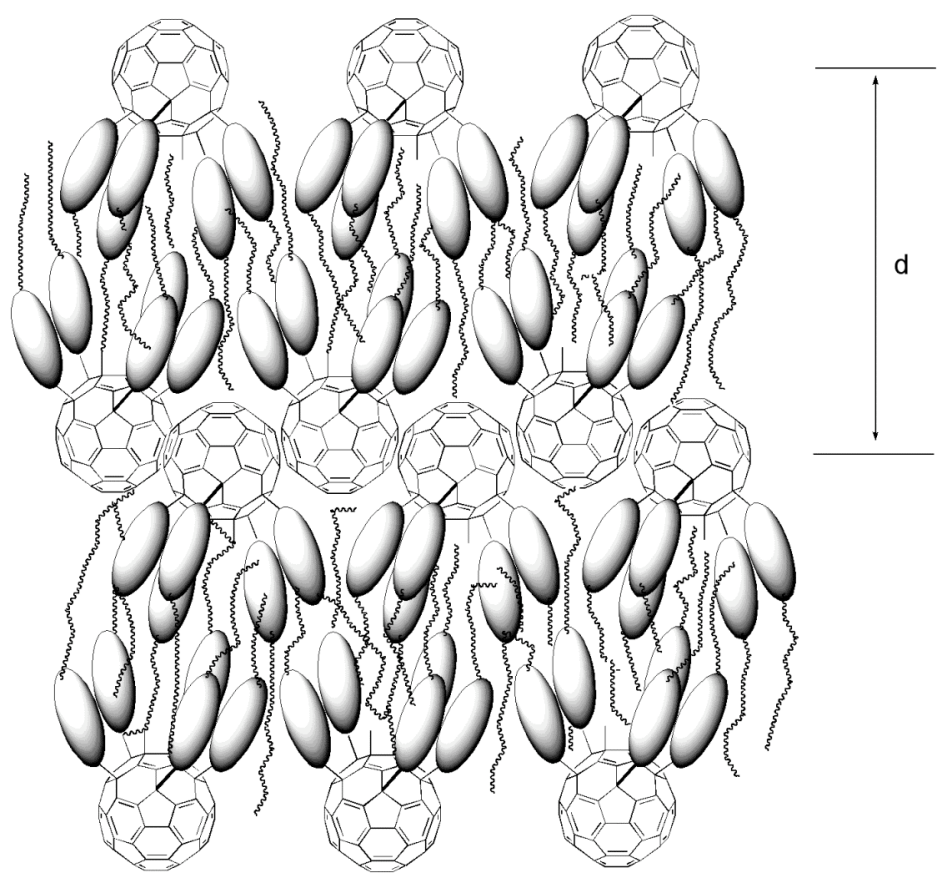

Figure S4. Schematic drawing of supramolecular organization in 4. 


\section{Optical Textures of Liquid Crystals}

(a)

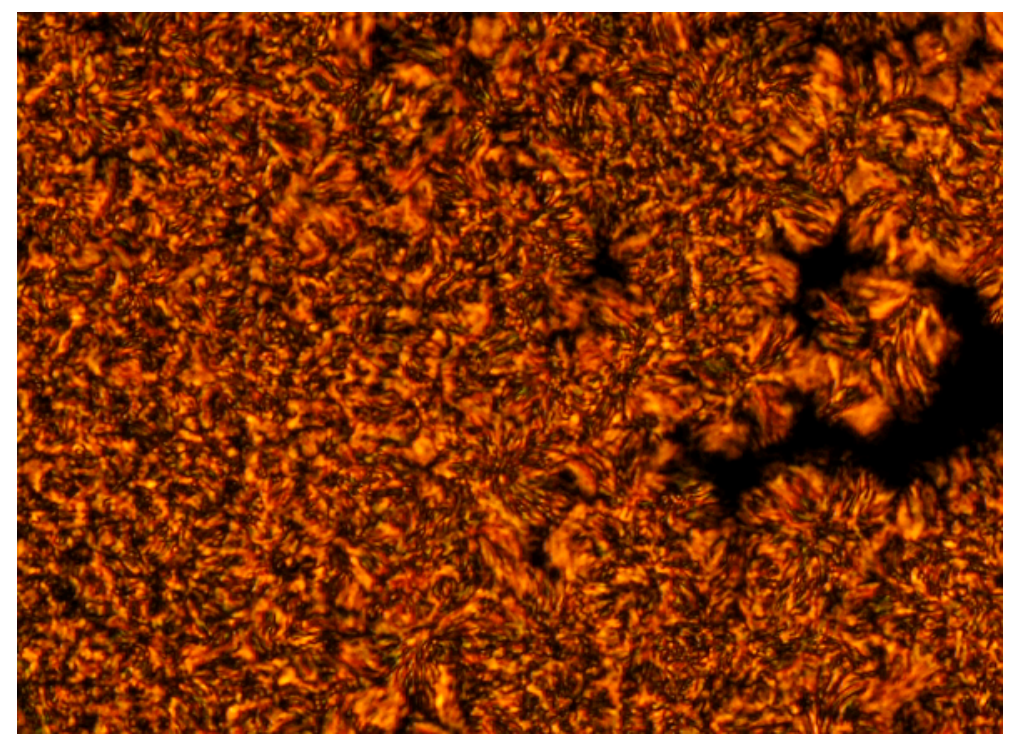

(b)

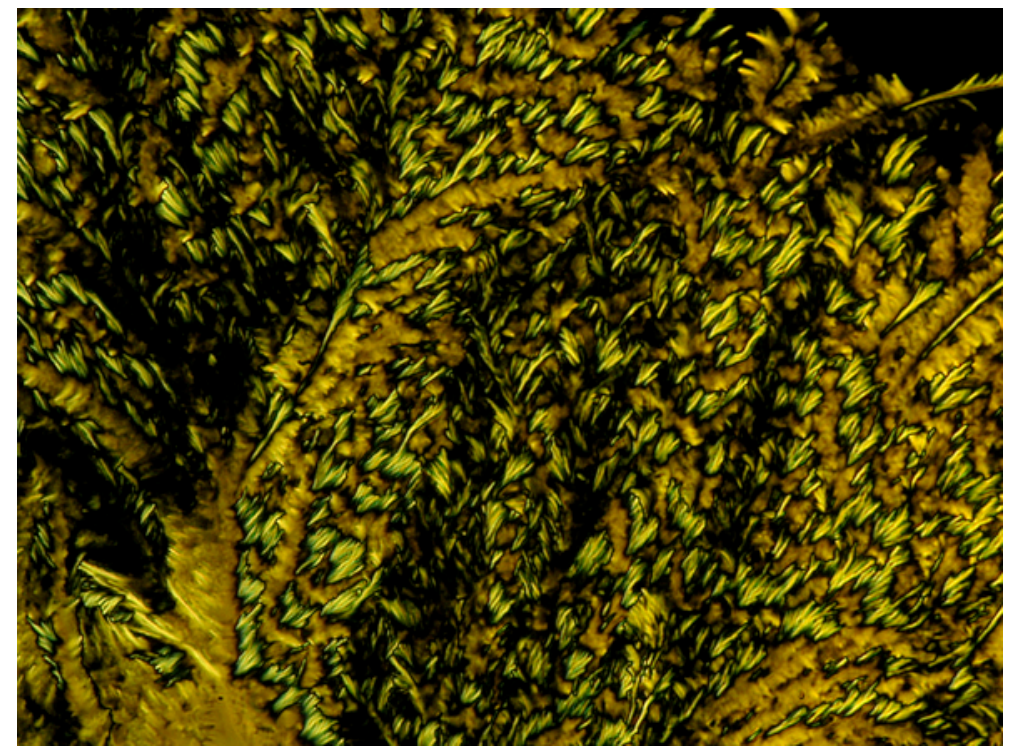

Figure S5. Optical texture of thermotropic liquid crystal $\mathrm{C}_{60} \mathrm{Me}\left[\mathrm{C}_{6} \mathrm{H}_{4} \mathrm{C} \equiv \mathrm{CSiMe}_{2}\left(n-\mathrm{C}_{10} \mathrm{H}_{21}\right)\right]_{5}$ (4) under optical polarized microscope. (a) Batonnet texture at $150{ }^{\circ} \mathrm{C}$ on the first heating. (b) Baton-like texture at $130{ }^{\circ} \mathrm{C}$ on cooling from isotropic liquid over $9 \mathrm{hrs}$ at a rate of $0.1{ }^{\circ} \mathrm{C} / \mathrm{min}$. 


\section{DSC Measurement of Liquid Crystals}

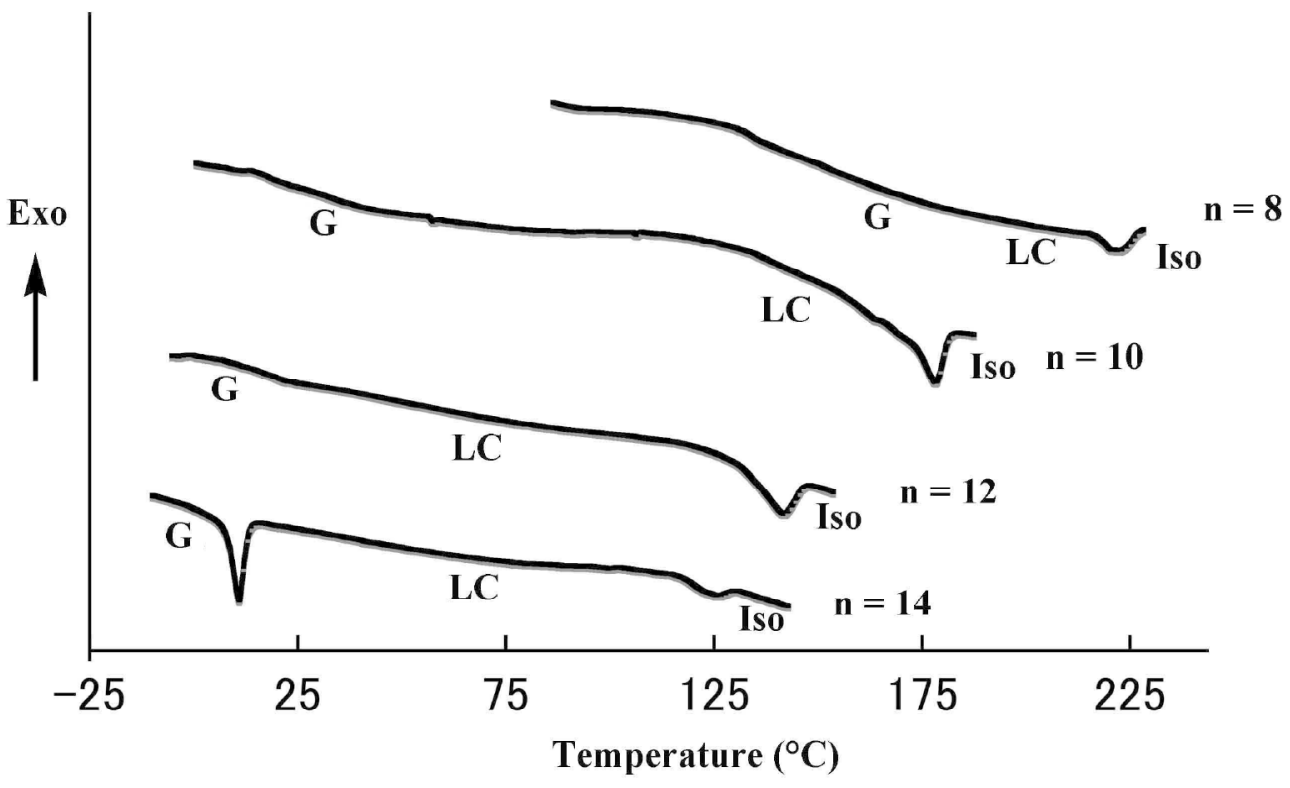

Figure S6. DSC curves on the second heating of liquid crystals 3, 4, 5, and 6 with alkyl chains number $\mathrm{n}=8,10,12$, and 14 , respectively.

Table S2. Phase transition behavior of the LC molecules 3-6 on the second heating ${ }^{a}$

\begin{tabular}{|c|c|c|c|c|c|c|}
\hline \multirow{2}{*}{$\begin{array}{c}\mathrm{C}_{60} \mathrm{Me}\left(\mathrm{C}_{6} \mathrm{H}_{4} \mathrm{C} \equiv \mathrm{CSiMe}_{2} \mathrm{C}_{\mathrm{n}} \mathrm{H}_{2 \mathrm{n}+1}\right)_{5} \\
\mathbf{3}\end{array}$} & \multirow{2}{*}{$\begin{array}{l}\mathrm{n} \\
8\end{array}$} & \multicolumn{5}{|c|}{$\begin{array}{l}\text { Phase transition temperature }\left({ }^{\circ} \mathrm{C}\right) \\
{[\text { Transition enthalpy }(\mathrm{kJ} / \mathrm{mol})]}\end{array}$} \\
\hline & & $\mathrm{G}$ & 140 & $\mathrm{Sm}$ & $\begin{array}{c}223 \\
{[1.4]}\end{array}$ & Iso \\
\hline 4 & 10 & $\mathrm{G}$ & 48 & $\mathrm{Sm}$ & $\begin{array}{r}178 \\
{[3.5]}\end{array}$ & Iso \\
\hline 5 & 12 & $\mathrm{G}$ & 19 & $\mathrm{Sm}$ & $\begin{array}{c}147 \\
{[3.1]}\end{array}$ & Iso \\
\hline 6 & 14 & $\mathrm{G}$ & $\begin{array}{c}11 \\
{[3.3]}\end{array}$ & $\mathrm{Sm}$ & $\begin{array}{c}126 \\
{[0.8]}\end{array}$ & Iso \\
\hline
\end{tabular}

${ }^{a}$ Abbreviations: G, glassy; Sm, smectic phase; Iso, isotropic liquid; Cr, crystalline. Transition temperature was determined by DSC at the rate of $10{ }^{\circ} \mathrm{C} \min ^{-1}$ and were taken at the maximum of each transition peak or mid temperature (for glassy/LC transition). 


\section{X-ray Diffraction Data for Liquid Crystals}
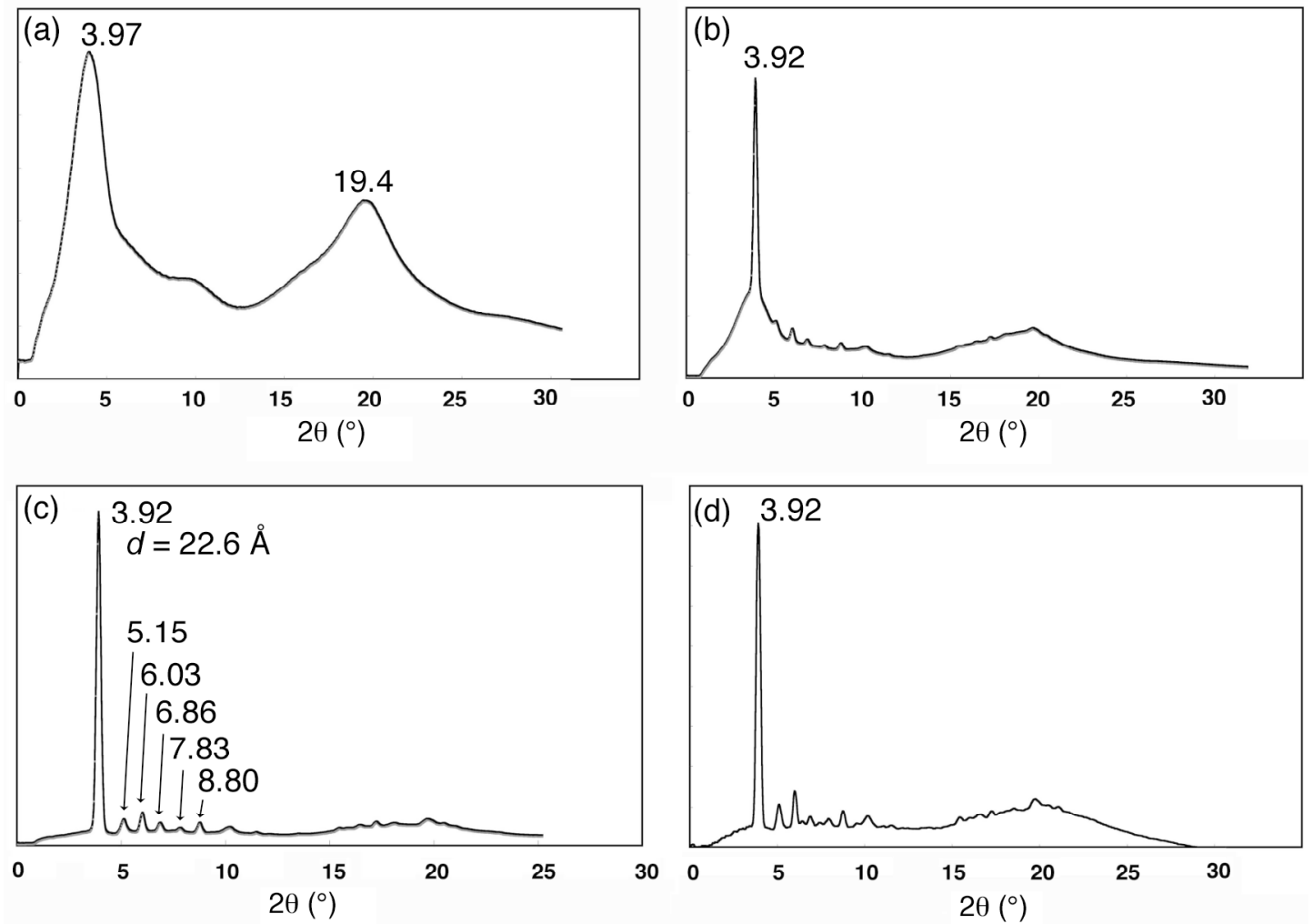

Figure S7. X-ray diffraction pattern of thermotropic liquid crystal $\mathrm{C}_{60} \mathrm{Me}\left[\mathrm{C}_{6} \mathrm{H}_{4} \mathrm{C} \equiv \mathrm{CSiMe}_{2}\left(n-\mathrm{C}_{10} \mathrm{H}_{21}\right)\right]_{5}(4)$. (a) At room temperature. (b) After heating at $130{ }^{\circ} \mathrm{C}$ for 2 hrs. (c) After heating at $130{ }^{\circ} \mathrm{C}$ for 7 hrs. (d) Standing at $130{ }^{\circ} \mathrm{C}$ for $10 \mathrm{hrs}$ after cooling from isotropic liquid $\left(190^{\circ} \mathrm{C}\right)$.
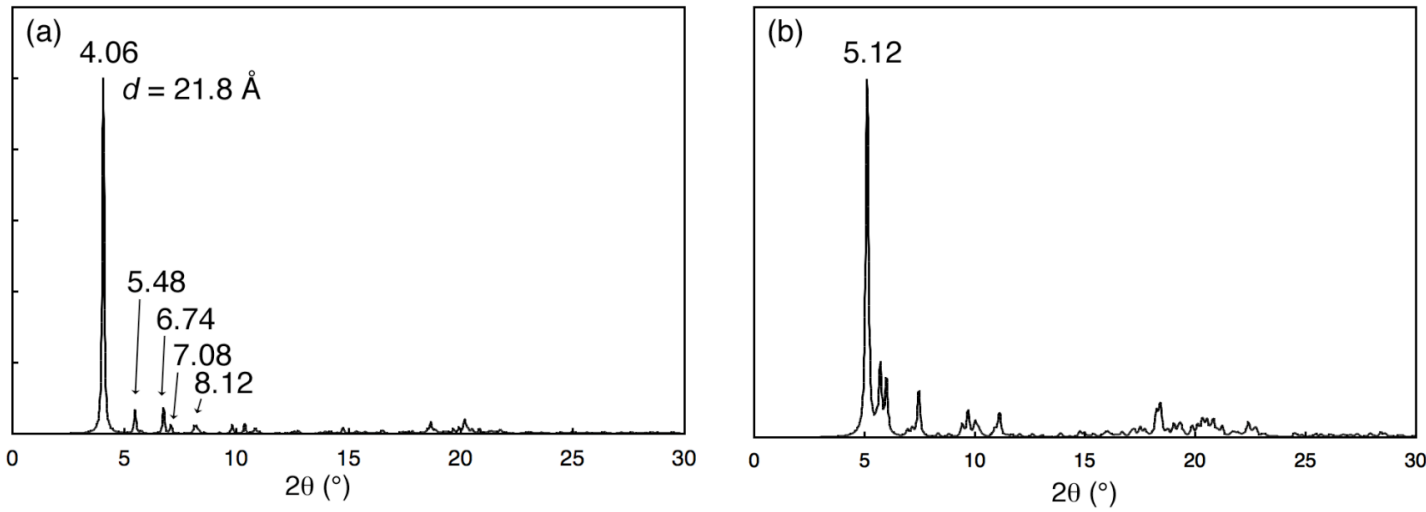

Figure S8. Simulated X-ray diffraction pattern based on single crystal X-ray crystallographic analysis. (a) Pattern for $\mathrm{C}_{60} \mathrm{Me}\left[\mathrm{C}_{6} \mathrm{H}_{4} \mathrm{C} \equiv \mathrm{CSiMe}_{3}\right]_{5}$ (toluene) $)_{2}$ (1-(toluene) $)_{2}$ ). (a) Pattern for $\mathrm{C}_{60} \mathrm{Me}\left[\mathrm{C}_{6} \mathrm{H}_{4} \mathrm{C} \equiv \mathrm{CSiMe}_{2}{ }^{n} \mathrm{Bu}\right]_{5}(\mathbf{2})$. 

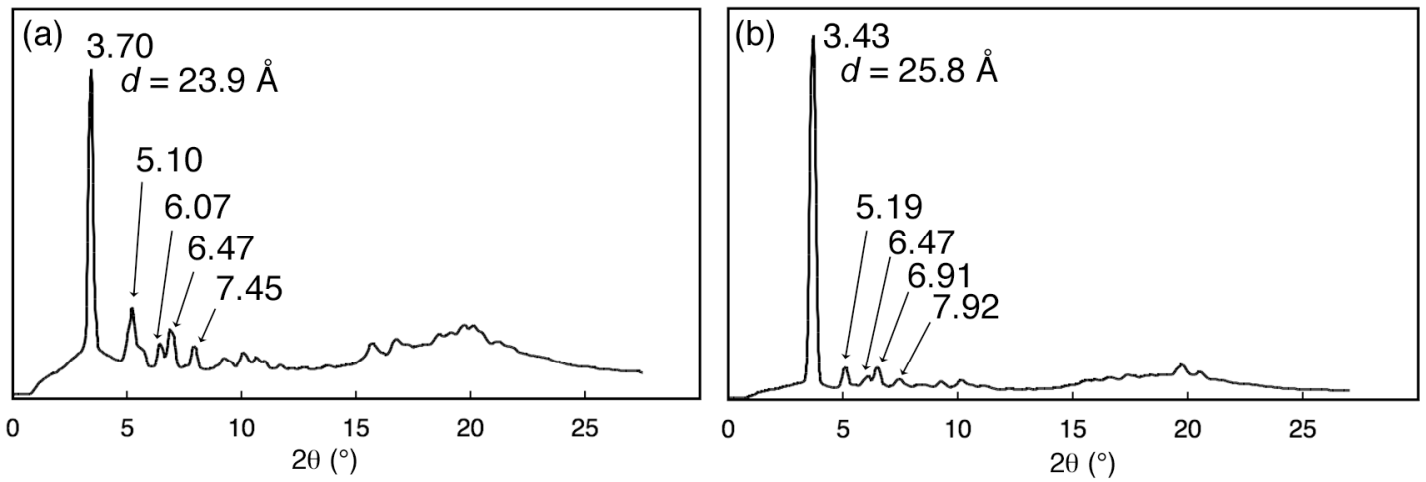

Figure S9. X-ray diffraction pattern at $100{ }^{\circ} \mathrm{C}$. (a) Compound 5. (b) Compound 6.

Table S3. Relationship between the interlayer distance and the atom length (n) of the alkyl chains on the molecules $\mathrm{C}_{60} \mathrm{Me}\left[\mathrm{C}_{6} \mathrm{H}_{4} \mathrm{C} \equiv \mathrm{CSiMe}_{2}\left(n-\mathrm{C}_{\mathrm{n}} \mathrm{H}_{2 n+1}\right)\right]_{5}$ with a layered structure. ${ }^{a}$

\begin{tabular}{ccc}
\hline $\mathrm{C}_{60} \mathrm{Me}\left(\mathrm{C}_{6} \mathrm{H}_{4} \mathrm{C} \equiv \mathrm{CSiMe}_{2} \mathrm{C}_{\mathrm{n}} \mathrm{H}_{2 \mathrm{n}+1}\right)_{5}$ & $\mathrm{n}$ & Interlayer distance $(\AA)$ \\
$\mathbf{1}$ & 1 & 22.4 \\
$\mathbf{2}$ & 4 & 16.2 \\
$\mathbf{3}$ & 8 & 20.5 \\
$\mathbf{4}$ & 10 & 22.6 \\
$\mathbf{5}$ & 12 & 23.9 \\
$\mathbf{6}$ & 14 & 25.8
\end{tabular}

${ }^{a}$ The interlayer distance of $\mathbf{1}$ and $\mathbf{2}$ was determined by X-ray analysis of their single crystals at $-120{ }^{\circ} \mathrm{C}$. The interlayer distance of $\mathbf{3}, \mathbf{4}, \mathbf{5}$, and $\mathbf{6}$ was determined by X-ray diffraction at $180^{\circ} \mathrm{C}, 130{ }^{\circ} \mathrm{C}, 110^{\circ} \mathrm{C}$, and $100{ }^{\circ} \mathrm{C}$ on the first heating process respectively.

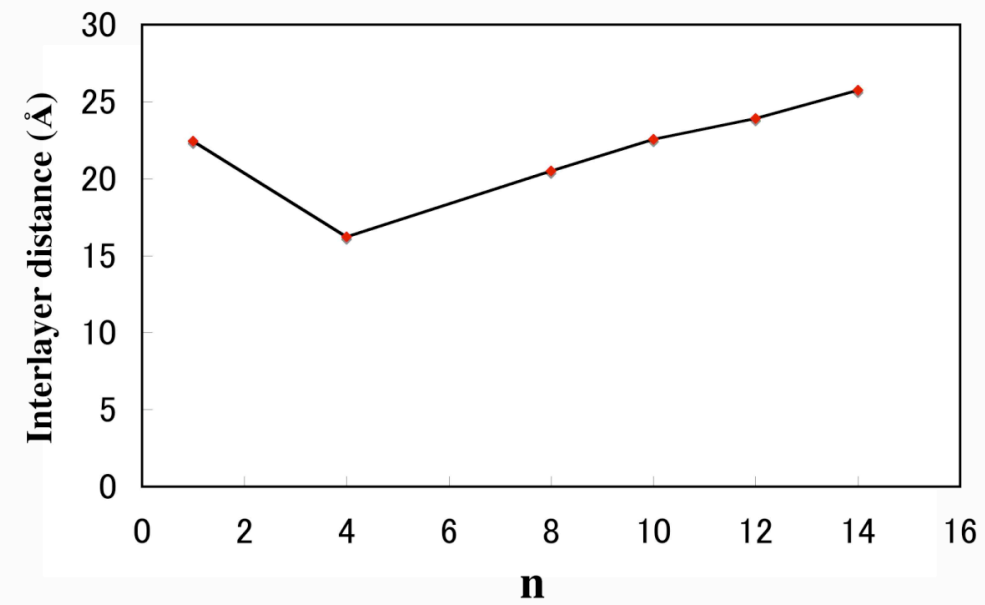

Figure S10. Relationship between the interlayer distance and the atom length (n) of the alkyl chains on the molecules $\mathrm{C}_{60} \mathrm{Me}\left[\mathrm{C}_{6} \mathrm{H}_{4} \mathrm{C} \equiv \mathrm{CSiMe}_{2}\left(n-\mathrm{C}_{\mathrm{n}} \mathrm{H}_{2 \mathrm{n}+1}\right)\right]_{5}$ with a layered structure. Note that the crystals of $\mathbf{1}$ contain toluene molecules in the interlayer space and hence may show a larger interlayer distance. Note that the crystals of $\mathbf{1}$ contain toluene molecules in the interlayer space and hence may show a larger interlayer distance. 